\title{
Eficacia y selectividad de Amicarbazone aplicado en diferentes dosis en caña panelera (Saccharum officinarum L.), en Güepsa, Santander
}

\section{Effectiveness and selectivity of Amicarbazone applied in different doses to panelera cane (Saccharum officinarum L.) in Güepsa, Santander}

Héctor Augusto Morcote Católico ${ }^{1}$

\begin{abstract}
Resumen
La eficacia y selectividad de los herbicidas están influenciadas por la cantidad, mecanismo y modo de acción del ingrediente activo (i.a.), así como por la influencia directa de los factores climáticos. En la presente investigación se evaluaron la eficacia y selectividad del herbicida Amicarbazone para controlar malezas en el cultivo de caña panelera, en el municipio de Güepsa, Santander. Se utilizó un diseño de bloques completos aleatorizados con seis tratamientos y cuatro repeticiones. Los tratamientos consistieron en diferentes dosis del herbicida (i.a. 700, 875, 1050, 1260, 1400 gr$\left.\mathrm{ha}^{-1}\right)$, aplicadas en el cultivo de caña panelera 40 días luego de la emergencia, con una infestación de malezas que superó el $65 \%$ de área efectiva. Se evaluó el porcentaje de control de las especies de malezas, eficacia del herbicida, control de malezas en función del peso, daño al cultivo, selectividad, número de macollas por planta y altura de las plantas. Los datos fueron tomados a los 15, 30, 45 y 60 días después de la aplicación (DDA). Las dosis evaluadas causaron algunos síntomas fitotóxicos al cultivo. Se detectó menor crecimiento en algunas características del cultivo, especialmente altura y número de macollas. Los tratamientos mostraron diferencias significativas
\end{abstract}

\begin{abstract}
The efficiency and selectivity of herbicides is directly influenced by the amount, mechanism and mode of action of the active ingredient (a.i), as well as the direct influence of climatic factors. In this context, the present study we evaluated the efficacy and selectivity of Amicarbazone herbicide to control weeds in sugarcane crop, in the municipality of Güepsa, Santander. The experimental design was randomized complete block with six treatments and four repetitions. Treatments consisted of different doses of the herbicide (a.i. 0, 700, 875, 1050, 1260, 1400 gr-ha $^{-1}$ ) applied in the sugarcane crop at 40 days after emergence, with a weed infestation exceeded $65 \%$ of effective area. The control percentage was evaluated for weed species, herbicide efficacy, weed control according to the weight, crop damage, selectivity, number of tillers per plant and plant height. The data were taken at 15, 30, 45 and 60 days after application (DAA). The evaluated doses of herbicides caused some phytotoxic symptoms to the crop. Slower growth was detected in some variables of the crop, especially the height and number of tillers. The treatments showed significant differences in overall control of weeds and effectiveness, although it is efficient,
\end{abstract}


en el control general de malezas y en la eficacia; a pesar de esto es eficiente, superando el $91 \%$ de control y el $92 \%$ de eficacia. El mejor tratamiento fue el de 875 gr-ha ${ }^{1}$ i.a., con $96,4 \%$ de actividad y grado de control, y $97,2 \%$ de eficacia.

Palabras clave: Control de malezas, Amicarbazone, Triazolinonas. (Fuente. USDA) exceeding $91 \%$ of control and $92 \%$ of efficacy. The best treatment a.i was $875 \mathrm{gr}^{-\mathrm{ha}^{-1}}$ with $96.4 \%$ of activity and control grade and $97.2 \%$ of efficacy.

Key words: Weed control, Amicarbazone, Triazolinone. (Source: USDA) 


\section{Introducción}

El cultivo de caña panelera en Colombia es de gran importancia socioeconómica, pues ocupa el séptimo lugar en extensión, después del café, el arroz, el maíz, el plátano, la palma de aceite y la caña de azúcar. Su cultivo, con predominio de explotación tradicional y artesanal, se constituye en sustento de muchos hogares (Fedepanela, 2010). En el país, la producción de caña panelera se concentra básicamente en los departamentos de Cundinamarca, Antioquia, Caldas, Santander y Boyacá (SIPA, 2010); según la Encuesta Nacional Agropecuaria de 2009, para este año el país contó con 203.919 hectáreas sembradas y con 154.117 hectáreas en edad productiva, ubicando al departamento de Santander como el de mayor número de hectáreas sembradas.

En la actualidad se busca minimizar los efectos de la interferencia de las malezas en el cultivo de caña panelera, con la aplicación de herbicidas en preemergencia y posemergencia; la aplicación de éstos para el control de malezas depende de las características químicas del producto, la dosis del herbicida, la humedad del suelo, las condiciones climáticas y las características intrínsecas del suelo (Sabbatini et al., 2004).

El Amicarbazone es el ingrediente activo de un herbicida del grupo químico de los Triazolinonas de aplicación preemergente y posemergente, que produce inhibición de la fotosíntesis en las malezas, en la fase II de la fotosíntesis, inhibiendo el transporte de electrones, la fijación del $\mathrm{CO}_{2}$ y la producción de ATP y $\mathrm{NADPH}_{2}$ (fase I de la fotosíntesis), que son esenciales para el crecimiento de las plantas (Toledo et al., 2009).

En Colombia es escasa la investigación sobre el control químico de malezas con Amicarbazone en el cultivo de caña panelera; como es una molécula nueva, el conocimiento del desempeño de este herbicida para el control de malezas en caña panelera puede brindar una alternativa para el manejo de este problema, ya que la información que se tiene de este producto ha sido generada en otros países, por lo que es necesario generarla regionalmente.

La producción de caña panelera se ve disminuida hasta en un $60 \%$ debido a las malezas y, en ocasiones, a la inoportuna aplicación de productos de síntesis química para su control, cuyo período de control no excede los 60 días, como en el caso de Hexazinona, Diuron y Ametrinas, haciendo necesaria una segunda aplicación, que origina daño mecánico al cultivo y permite que malezas en estados adelantados se multipliquen y prevalezcan (Esqueda, 2005). Una de las alternativas del control de malezas en caña panelera en posemergencia es la aplicación de Amicarbazone (Dinamic ${ }^{\circledR} 70$ WG), que pertenece a la familia de las Triazolinonas (inhibidor del fotosistema II), sin embargo, se han reportado efectos fitotóxicos (Aguilar et al., 2007).

Las malezas se encuentran entre los factores más limitantes en la producción de cultivos, ya que causan daños directos e indirectos por la competencia de luz, agua y nutrientes; pueden disminuir la calidad de la cosecha y ser hospederas de insectos-plagas y de enfermedades, y, además, producir compuestos alelopáticos que afectan el crecimiento normal del cultivo.

\section{Materiales y Métodos}

Ubicación. El ensayo se realizó en el municipio de Güepsa, Santander, ubicado a $6^{\circ}$ 02' latitud Norte y $73^{\circ} 35^{\prime}$ longitud Oeste y a $1400 \mathrm{~m}$ de altura. La topografía es ondulada, la precipitación anual de la zona es de $1600-1800 \mathrm{~mm}$ año y la humedad relativa es del $85 \%$. El cultivo de caña estaba sembrado con una densidad de 120.000 plantas $\cdot$ ha $^{-1}$. La variedad utilizada es RD 75-11, proveniente de plantilla, a la que no se le ha aplicado fertilización o riego. La cobertura general del lote por las malezas es del $65 \%$. Se ubicaron aleatoriamente 2 cuadros de $50 \times 50 \mathrm{~cm}$, en cada unidad experimental.

Aplicación. Se realizó la aplicación en cada parcela en posemergencia del cultivo, a los 40 días luego de la emergencia; se empleó una aspersora de espalda con capacidad de $20 \mathrm{~L}$, provista de una boquilla "Teejet" 8004; se calibró la descarga para aplicar $1400 \mathrm{~L} \cdot$ há $^{-1}$; se pesó el producto Dinamic ${ }^{\circledR}$ 70 WG con una balanza eléctrica para preparar $100 \mathrm{~L}$ de mezcla de cada dosis. Las aplicaciones se realizaron entre las 9 a.m. y 3 p.m.

\section{Variables evaluadas}

- Parámetros poblacionales básicos de las malezas. Se utilizó el método de la cuadrata (Braun-Blanquet, 1979), lanzando 10 veces en forma aleatoria un marco de $0,5 \times 0,5$ metros en el área general del cultivo; se recogió el material 
vegetal que quedó dentro del marco, se clasificó por especie, se contó el número de individuos por especie y se determinó frecuencia, densidad y dominancia.

- Porcentaje del control de malezas (PCM). Se realizó en función del área del suelo cubierta por las arvenses; para esto se observó el área efectiva de cada parcela y el área de infestación, lo que permitió calcular el control relativo (comparados con el testigo total enmalezado) de los tratamientos. Se empleó la escala de evaluación de porcentaje de control de malezas (Tabla 1) y se le asignó un valor porcentual de control a cada U.E. También se observó el área interna de cada cuadro y se determinó el área de cobertura general y por grupo de especies presentes (ALAM, 1974).

- Eficacia. Se realizó determinando el número de individuos vivos por especie en cada uno de los cuadrados ubicados en las parcelas, y se comparó con los individuos encontrados en el testigo de cada bloque. Se determinó el porcentaje de eficacia utilizando la ecuación propuesta por Henderson-Tilton (Rosset y Siman, 1990):

$$
E f=(1-\mathrm{Td} / \mathrm{Cd} * \mathrm{Ca} / \mathrm{Ta}) * 100
$$

Dónde:

Ta = Infestación en parcela tratada antes del tratamiento; $\mathrm{Td}=$ Infestación en parcela tratada después del tratamiento; $\mathrm{Ca}=$ Infestación en parcela Testigo antes del tratamiento; $\mathrm{Cd}$ = Infestación en parcela testigo después del tratamiento.

- Tolerancia del cultivo. Se realizaron observaciones al cultivo antes y luego de la aplicación, según la metodología propuesta por ALAM (1974) para medir la escala de daño al cultivo. Esta evaluación se hace en forma general en cada parcela, comparándola con el testigo total enmalezado o sin aplicación, lo cual se fundamenta en los cambios que se suceden en el cultivo por la aplicación del herbicida; luego, se le asigna un porcentaje (\%) por unidad experimental a cada tratamiento.

- Selectividad. Se efectuó revisando detenidamente las 10 plantas marcadas en los surcos centrales y haciéndole una evaluación a cada una para así determinar el daño, según la escala para la evaluación de daños ya mencionada, asignándole a cada planta un Valor Categoría, y a cada grupo de plantas, un Porcentaje de Infestación. Se determinó el porcentaje de selectividad con la ecuación de Townsend-Heuberger modificada.

$$
\% S=\frac{\sum_{0}^{\mathrm{i}}(\mathrm{n} * \mathrm{v}) * 100}{\mathrm{i} *-\mathrm{N}}+100
$$

Dónde:

$\mathrm{v}=$ Valor de la categoría; $\mathrm{i}=$ valor de la categoría más alta; $\mathrm{n}$ = número de plantas en cada categoría; $\mathrm{N}=$ total de plantas investigadas.

- Evaluación macollas por planta. Se contó el número de macollas en cada sitio, en las 10 plantas marcadas a medida que pasa el tiempo.

- Evaluación altura de plantas. Se midió la altura de cada planta, tomándola desde la base del suelo hasta el extremo superior de la yema apical. Esto en la macolla marcada desde el día cero.

Diseño experimental. El diseño experimental utilizado fue de bloques completos al azar, con 6 tratamientos y 4 repeticiones. Tratamiento 0 : i.a. cero $\mathrm{gr} \cdot \mathrm{ha}^{-1}$; Tratamiento 1: i.a. $700 \mathrm{gr} \cdot \mathrm{ha}^{-1}$; Tratamiento 2: i.a. $875 \mathrm{gr} \cdot \mathrm{ha}^{-1}$; Tratamiento 3: i.a. $1050 \mathrm{gr} \cdot \mathrm{ha}^{-1}$; Tratamiento 4: i.a. $1660 \mathrm{gr} \cdot \mathrm{ha}^{-1}$; Tratamiento 5: i.a. $1400 \mathrm{gr} \cdot \mathrm{ha}^{-1}$. El tamaño de la parcela fue de 8 surcos por 10 metros de largo; el área efectiva se tomó de los seis surcos centrales y sus siete calles próximas. En total fueron 24 unidades experimentales.

Método estadístico. Se realizó un análisis de varianza convencional correspondiente al diseño de bloques completos al azar; también se sometieron a la prueba de comparación de medias de Tukey $(P \leq 0.05)$ con un nivel de confiabilidad de 95\% mediante el paquete estadístico SAS 8.1.

\section{Resultados y Discusión}

El material fue recolectado de acuerdo con la metodología preestablecida, evidenciando la presencia de ciperáceas, gramíneas y hojas anchas. Se identificaron los nombres comunes y científicos. Se encontró que las malezas con menor presencia son las ciperáceas (Tabla 1); con presencia media, las gramíneas (Tabla 2), y las más abundantes, las hojas anchas (Tabla 3); esto 
haciendo una comparación de densidad de plantas, dominancia y frecuencia. Se pudo establecer que no siempre la maleza con mayor dominancia es la más abundante.

La distribución de las especies observadas concuerda de manera general con lo reportado; hay prevalencia de gramíneas, ciperáceas y algunas hojas anchas, especies indicadas en plantaciones de caña de azúcar en Venezuela y Cuba, destacando que su presencia se manifiesta en suelos de mayor tradición cañera y medianamente fértiles, tal como corresponde a la región estudiada (Valle et al., 2000).

Tabla 1. Parámetros poblacionales básicos ciperáceas

\begin{tabular}{|c|c|c|c|c|}
\hline Nombre común & Nombre científico & $\begin{array}{c}\text { Frecuencia } \\
(\mathbf{\%})\end{array}$ & $\begin{array}{c}\text { Densidad } \\
\left.\text { (plantas } \mathbf{~ m}^{\mathbf{2}}\right)\end{array}$ & Dominancia (\%) \\
\hline Cortadera & Cyperus digitatus & 30 & 7,2 & 4,73 \\
\hline Cortadera & Cyperus ferax & 50 & 8 & 5,26 \\
\hline Coquito & Cyperus rotundus & 10 & 1,2 & 0,78 \\
\hline
\end{tabular}

Tabla 2. Parámetros poblacionales básicos gramíneas

\begin{tabular}{|c|c|c|c|c|}
\hline Nombre común & Nombre científico & $\begin{array}{c}\text { Frecuencia } \\
\mathbf{( \% )}\end{array}$ & $\begin{array}{c}\text { Densidad } \\
\left(\mathbf{P l a n t a s} \mathbf{~ m}^{\mathbf{2}}\right)\end{array}$ & $\begin{array}{c}\text { Dominancia } \\
\mathbf{( \% )}\end{array}$ \\
\hline Pasto para & Brachiaria decumbens & 50 & 10 & 6,57 \\
\hline Yaragua & Paspalum bonplandianum & 50 & 9,2 & 6,05 \\
\hline Xyris & Xyris sp & 20 & 3,6 & 2,36 \\
\hline
\end{tabular}

Tabla 3. Parámetros poblacionales básicos hoja ancha

\begin{tabular}{|c|c|c|c|c|}
\hline Nombre común & Nombre científico & $\begin{array}{c}\text { Frecuencia } \\
\text { (\%) }\end{array}$ & $\begin{array}{c}\text { Densidad } \\
\text { (Plantas } \mathbf{~ m}^{\mathbf{2}}\end{array}$ & $\begin{array}{c}\text { Dominancia } \\
\mathbf{( \% )}\end{array}$ \\
\hline Ulluco & Ullucus tuberosus & 30 & 34,8 & 22,89 \\
\hline Frijolillo & Desmodium orinicense & 70 & 13,2 & 8,68 \\
\hline Suelda con suelda & Tripogandra encolea (Diels) & 30 & 8 & 5,26 \\
\hline
\end{tabular}

En general, las malezas ocuparon el 70\% del área total del lote, lo cual reafirma el hecho de que son un problema que requiere de un control radical; máxime cuando el cultivo está en su etapa inicial, que es cuando requiere buena disponibilidad de nutrientes y espacio suficiente para poder iniciar su desarrollo de forma exitosa. La presencia de malezas, en algunos casos por la presión ejercida sobre el cultivo, hace que éste detenga su ciclo vegetativo y dé paso a la proliferación de malezas. El estado de desarrollo de las malezas ciperáceas y gramíneas a partir de los 40 días con malezas ha mostrado una relación directa con la disminución del rendimiento agrícola de la caña (Arias et al., 1999).

Porcentaje control de malezas (PCM). En los tres tipos de malezas (ciperáceas, gramíneas y hojas anchas), el porcentaje de control de la cobertura fue mayor a medida que el tiempo aumentó, pero se observó un control más alto entre los 15 y 30 días después de la aplicación (DDA); este

Eficacia y selectividad de Amicarbazone aplicado en diferentes dosis en caña panelera

(Saccharum officinarum L.), en Güepsa, Santander - 51 
comportamiento se ajustó a una función cuadrática. Se presentaron diferencias altamente significativas $(p \leq 0,01)$. Las malezas de hoja ancha fueron las que presentaron mayor control, seguidas de las gramíneas y, luego, de las ciperáceas.

El mayor control encontrado en las hojas anchas se debe a que éstas son las plantas con mayor densidad y dominancia, y que por características influyen en la actividad del herbicida, por ejemplo, la edad y porte: las plantas jóvenes son más sensibles que las adultas (porque poseen menos barreras físicas y más células dividiéndose activamente), y las anuales más que las perennes; además de esto, el herbicida es específico para el control de ellas. La morfología de éstas también influye en la selectividad: inclinación de la hoja, grosor de la cutícula, pilosidad, localización de los meristemos (por ejemplo, en las ciperáceas y gramíneas están a ras de suelo, más protegidos que en las hojas anchas) y el sistema radicular; las especies que explotan la capa más superficial del suelo son más sensibles a los herbicidas que las que profundizan más (De Prado et al.,1999). La fisiología y bioquímica de cada planta también influyen en la selectividad (capacidad de translocar el herbicida, de detoxificarlo o, bien, de sucumbir ante él.). Por otro lado, las hojas anchas son las plantas más grandes y tienen un área de cobertura mayor por la forma de las hojas y el hábito rastrero en la mayoría de los casos, y las gramíneas y ciperáceas crecen en conglomerados y profundizan más su raíz.

El control ejercido por el herbicida sobre el área de cobertura de la totalidad de las malezas mostró un comportamiento creciente, caracterizado por un mayor porcentaje de control entre los 15 y 30 DDA; a los 60 DDA se observó un porcentaje de control superior al $90 \%$ con todas las dosis del herbicida, excepto el testigo. Se encontraron diferencias estadísticas en todas las épocas de muestreo entre la dosis 0 y las demás dosis, pero entre estas últimas no hubo; no obstante, la mejor dosis fue la de $875 \mathrm{~g} \cdot \mathrm{ha}^{-1} \mathrm{de}$ Amicarbazone.

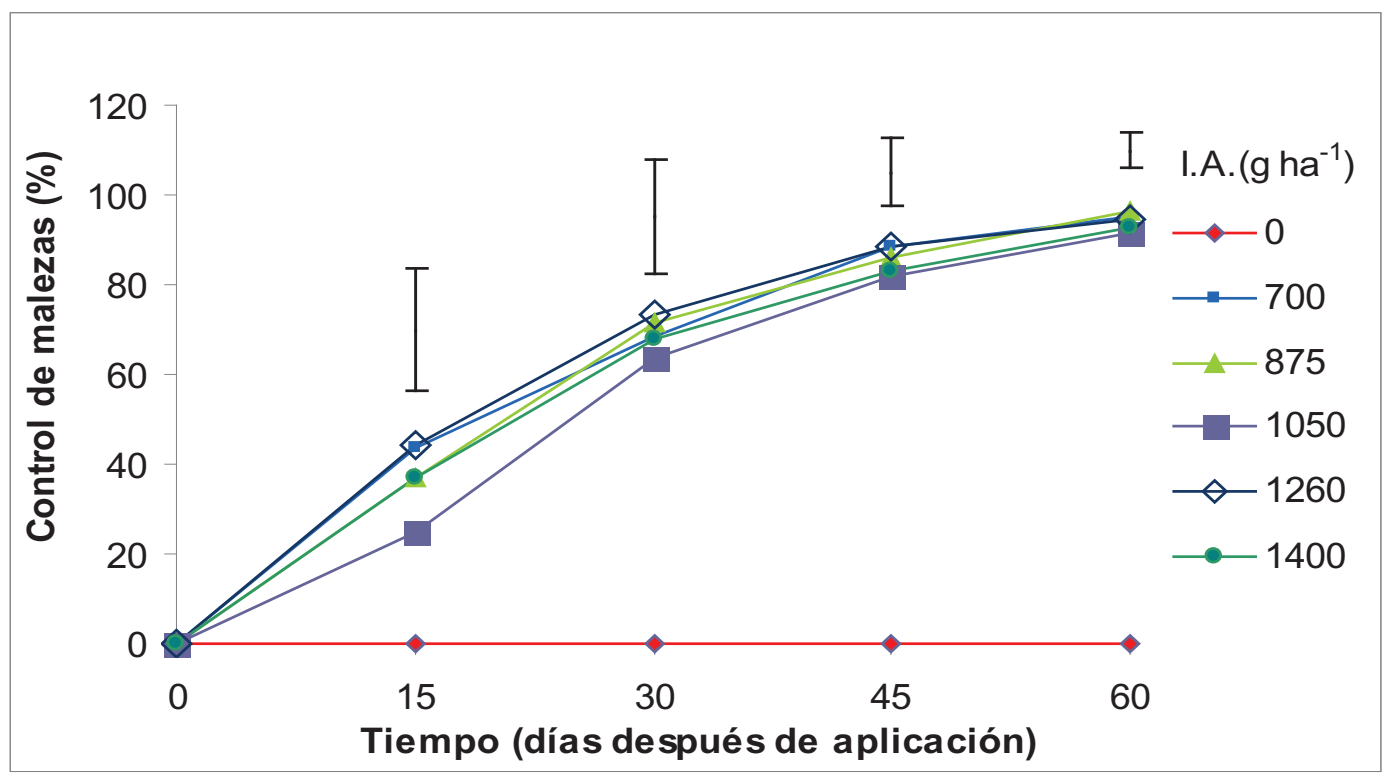

Figura 1. Efecto de diferentes dosis del herbicida Amicarbazone sobre el control del área de cobertura de la totalidad de las malezas en caña panelera.

En la evaluación hubo control efectivo del área de cobertura de las malezas en general a partir del día 45 DDA; no se evidenció diferencia significativa entre tratamientos, a excepción del control T0 en el día 45 DDA. El \% de actividad fue progresivo y se llegó a controlar hasta un 95\% de las malezas; el grado de control en la mayoría de los tratamientos fue excelente; hubo diferencias significativas entre tratamientos al día 60 DDA; a pesar de esto, el control en general fue excelente.

Eficacia. El porcentaje de eficacia en el control de la totalidad de las malezas fue mayor conforme aumentó el tiempo después de la aplicación. Respecto a cada uno de los tres tipos de malezas, la eficacia aumentó, siguiendo una función 
cuadrática, con mayor aumento hasta los 60 DDA. Aunque no se presentaron diferencias estadísticas en la eficacia del control entre tipos de malezas, al final hubo mayor eficacia en ciperáceas, y menor en las malezas de hoja ancha.

El porcentaje de eficacia en el control total de malezas aumentó hasta los 60 DDA, pero hubo un aumento más representativo de los 0 a los 30 DDA; después, el aumento fue muy inferior. Después de la aplicación del herbicida se presentaron diferencias estadísticas hasta los 60 DDA entre todas las dosis y el testigo. Aunque la aplicación de Amicarbazone en cualquiera de las dosis evaluadas mostró una eficacia muy alta, el mayor valor se obtuvo con i.a. $875 \mathrm{~g} \cdot \mathrm{ha}^{-1} \mathrm{de}$ Amicarbazone.
El porcentaje de eficacia en el control general de las malezas en las unidades experimentales por cada tratamiento no mostró diferencias significativas hasta el día 30 DDA, a excepción del tratamiento control. A partir del día 45 DDA, el \% de eficacia aumentó paulatinamente para todos los tratamientos, mostrando diferencias significativas entre tratamientos y el testigo. Al día 60 DDA, la mejor eficacia se obtuvo con el tratamiento i.a. 875 gr-ha $^{-1}$, con un $97,2 \%$ de eficacia para la generalidad de las malezas; el tratamiento de menor eficacia fue el i.a. 1259 gr-ha-1 $^{-1}$, con una eficacia de $92,3 \%$. Al final de la toma de datos, el \% de eficacia para todos los tratamientos, a excepción del T0, fue superior al 90\%; en general, la eficacia es buena para todos éstos, lo que demuestra un efecto alto de las diferentes aplicaciones sobre las malezas.

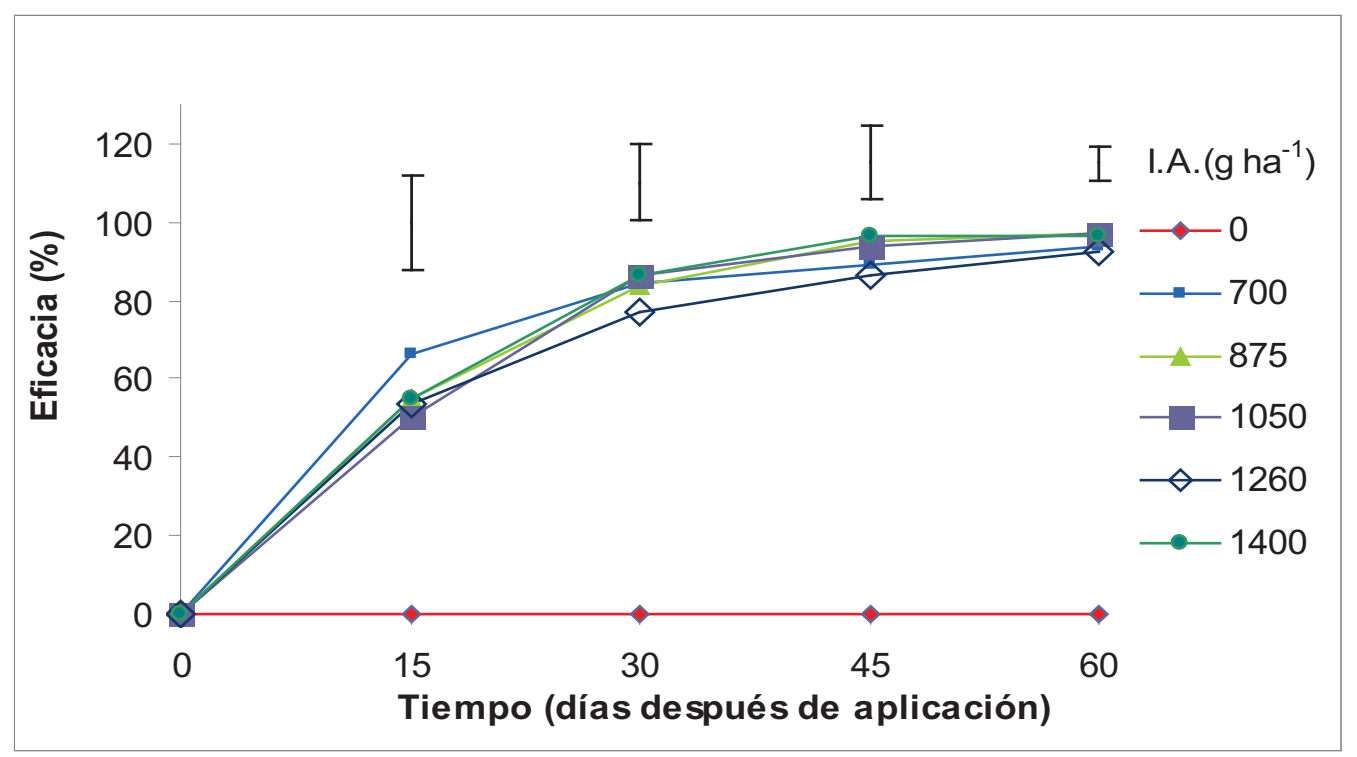

Figura 2. Porcentaje (\%) de eficacia del herbicida Amicarbazone en diferentes dosis sobre el control de la totalidad de las malezas en caña panelera.

Tolerancia del cultivo cualitativo. La aplicación del herbicida Amicarbazone generó daño en las plantas de caña, y fue estadísticamente superior al testigo, pero no fue significativamente diferente entre las dosis del herbicida; sin embargo, este daño no aumentó de forma representativa después de los 15 DDA, no obstante, a los 60 DDA el mayor daño se causó con la dosis de $1400 \mathrm{~g} \cdot \mathrm{ha}^{-1}$ de Amicarbazone. 


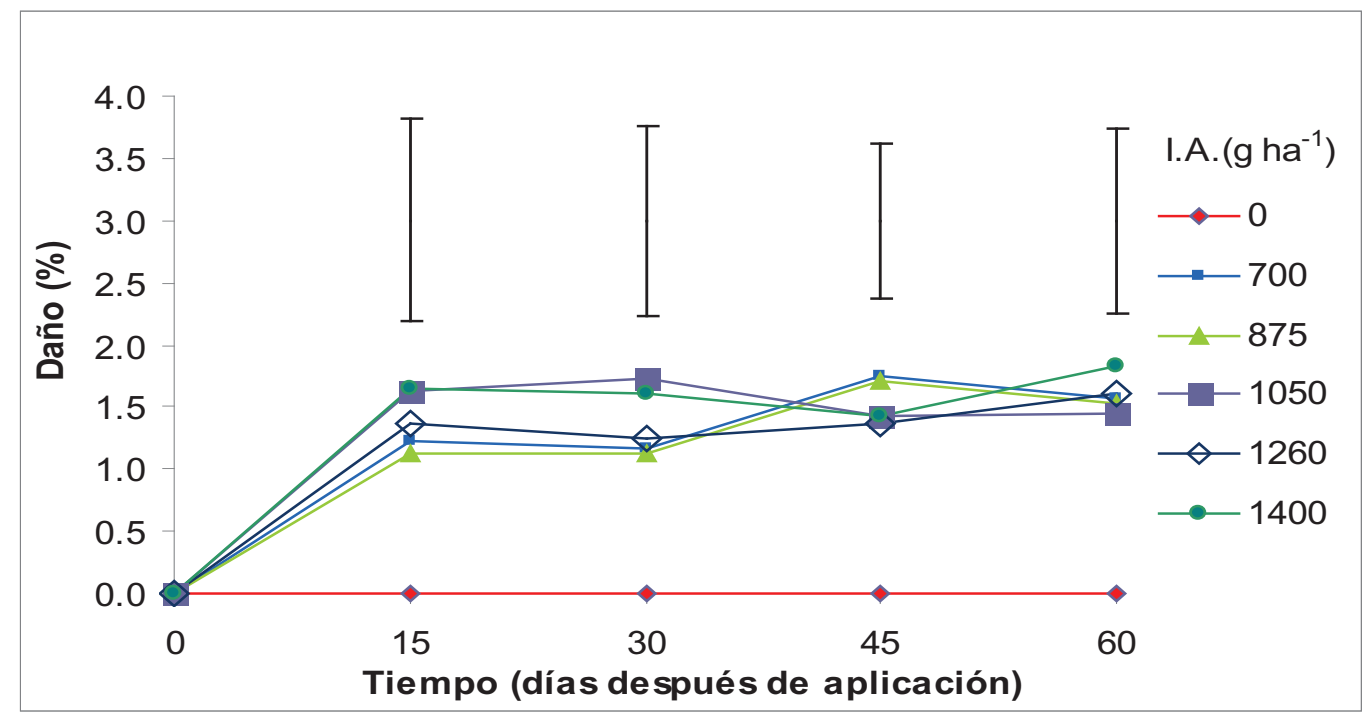

Figura 3. Tolerancia de la caña panelera al efecto de diferentes dosis del herbicida Amicarbazone.

Para todos los tratamientos no se evidenció daño aparente en el cultivo, lo cual puede probar la alta selectividad del producto al cultivo de caña, independiente de las dosis que se evaluaron en este ensayo. A pesar de haber diferencias significativas entre el T0 y los demás tratamientos, no se originó visualmente daño al cultivo. Las aplicaciones con 1050 gr.i.a-ha-1 fueron las que más toleró el cultivo; la media de daño no superó el $1.5 \%$, denominado con: Ningún daño, y descripción del daño: Ningún efecto, con apariencia similar al testigo.

Selectividad. El porcentaje de selectividad del herbicida con respecto al testigo en las plantas de caña disminuyó hasta los 15 DDA, y después permaneció estable; sólo después de los 30 DDA en adelante se presentaron diferencias estadísticas entre las dosis del herbicida y el testigo. A los 60 DDA se presentó menor selectividad con 1400 $\mathrm{g} \cdot \mathrm{ha}^{-1}$ de Amicarbazone.

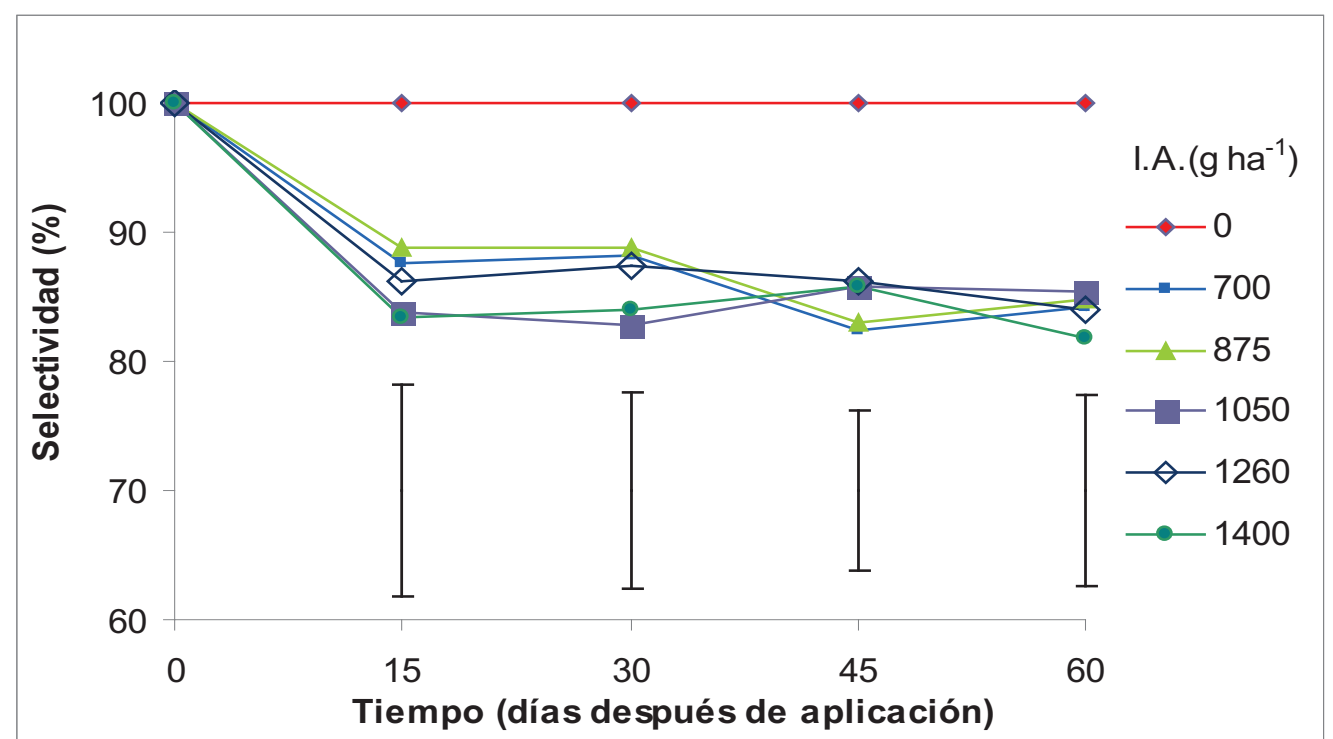

Figura 4. Porcentaje de selectividad de la caña panelera al efecto de diferentes dosis del herbicida Amicarbazone. 
A pesar de no haberse apreciado daño visualmente, el porcentaje de selectividad al cultivo fue decreciendo con el paso del tiempo. A los 15 DDA, la selectividad estaba alrededor del $83,5 \%$ para el tratamiento i.a. $1400 \mathrm{gr} \cdot \mathrm{ha}^{-1}$, y $87,5 \%$ para el tratamiento i.a. 700 gr-ha- $^{-1}$; para el día 60 DDA, el tratamiento T5 tenía un selectividad del $81 \%$, y el T1, del $84,25 \%$; el mejor tratamiento a esta fecha fue el i.a. $1050 \mathrm{gr}^{-\mathrm{ha}^{-1}}$, con $85,5 \%$ de selectividad. En general se presentó una leve clorosis y un retardo en el crecimiento, ya que los valores de selectividad oscilan entre el $90 \%$ y el $81 \%$, según tabla para evaluación de daños.

Macollas por planta. El número de macollas fue aumentando de forma continua a través del tiempo, con un más marcado entre los 45 y 60 DDA. No se observaron diferencias estadísticas entre tratamientos en ninguna época de muestreo; a pesar de esto, hasta los 45 DDA el testigo absoluto generó el mayor número de macollas, pero a los 60 DDA esta respuesta se observó con $1260 \mathrm{~g} \cdot \mathrm{ha}^{-1}$ de Amicarbazone.

Se evidenció un limitación en la aparición de macollas; el tratamiento que más macollas tuvo al día 60 DDA fue el de i.a. 0 gra-ha $^{-1}$, con un promedio de 5,175 macollas por sitio; el que menos macollas tuvo fue el tratamiento i.a. 700

\section{Literatura citada}

Asociación Latinoamericana de Malezas ALAM. (1974). Recomendaciones sobre unificación de los sistemas de evaluación en ensayos de control de malezas. ALAM; 1(1), 35-38.

Aguilar-Mariscal, I., Arriaga-Bayardi, R. (2007). Pegaso (Amicarbazone) en dos aplicaciones fraccionadas para el control de malezas en maíz. Iguala, México D. F, 32 p.

Arias, G., Salazar, J. D. (1999). Período crítico de inferencia por malezas en el cultivo de caña de azúcar (Saccharum officinarumk), ciclo planta, variedad pindar, en el ingenio Quebrada Azul, San Carlos, Costa Rica. Congreso Nacional Agronómico.

Braun-Blanquet, J. (1979). Fitosociología, bases para el estudio de las comunidades vegetales. España.

De-Prado, R., Osuna, M. D. (1999). Resistencia a herbicidas. Detección en campo y laboratorio. Congreso 1999 de la semilla. Logroño, España. pp. 435-440. gr-ha-1 ${ }^{-1}$ con 4,4 macollas. En general, la aparición de macollas se puede deber al momento de la aplicación del producto, que varió, ya que los tratamientos con menor i.a. gr-ha $^{-1}$ se aplicaron primero, y los de menor cantidad, en la tarde. Se puede considerar la dosis de i.a. 1400 gr-ha $^{-1}$ como un límite de cantidad de producto por aplicación, ya que tiene un promedio de 4,8 macollas por planta.

Altura de plantas. La altura de las plantas tuvo un leve aumento hasta los 30 DDA, y luego aumentó de forma representativa hasta los 60 DDA. No se presentaron diferencias estadísticas en ningún muestreo, pero la mayor altura de las plantas de caña se obtuvo con el testigo absoluto, y la menor, a los 60 DDA, con la aplicación de i.a. $1050 \mathrm{gr} \cdot \mathrm{ha}^{-1}$ de Amicarbazone.

Se marcó una leve disminución en el crecimiento de las plantas a las que se aplicó con cualquier dosis; el testigo T0 tuvo una altura promedio de $24,47 \mathrm{~cm}$, a diferencia de la mejor dosis, que fue $20,9 \mathrm{~cm}$ de altura. Hubo diferencias significativas entre el testigo y la mayoría de los tratamientos; los tratamientos con dosis más altas, i.a. 1260 gaha $^{-1}$, e i.a 1400 gr-ha $^{-1}$, fueron los que más altura promedio alcanzaron. El mejor tratamiento fue el i.a. 1260 gr-ha $^{-1}$, alcanzó una altura de $23 \mathrm{~cm}$, a pesar de esto, está por debajo del T0 control.

Esqueda, E. (2005). Determinación de la época de aplicación del herbicida Amicarbazone en caña de azúcar de riego. Instituto Nacional de Investigaciones Forestales, Agrícolas y Pecuarias. Apartado Memoria XXVI Congreso Nacional de la Ciencia de la Maleza. Veracruz. Cd. Victoria; p. 64-69.

Fedepanela 2010. Producción año (2009). Bogotá D. C.: Fondo Nacional de la Panela. 5 p.

Rosset, P. Y., Simán, G. (1990). Curso de muestreo para la evaluación de ensayos de campo en fitoprotección. $75 \mathrm{p}$.

Sabbatini, M. R., Irigoyen, J. H., Vernavá, M. N. (2004). Parte VIII: Aplicaciones capítulo 11: Estrategias para el manejo integrado de malezas: problemática, resistencia a herbicidas y aportes de la biotecnología. En: Biotecnología y mejoramiento vegetal. 25-34. Sistema de Información Panelero SIPA. (2010). Encuesta Nacional Panelera (realizada durante los años 2004-2005 y validada 
durante los años 2008-2009). Bogotá D.C.: Sistema de Información Panelero (SIPA). 3 p.

Toledo, R. E. B., Perim, L., Negrisoli, E., Corrêa, M. R., Carbonari, C. A., Rossi, C. V. S., Velini, E. D. 2009. Eficácia do herbicida amicarbazone aplicado sobre a palha ou no solo no controle de plantas daninhas na cultura da cana-deaçúcar, en planta daninha, Viçosa-Mg, 27(2), 319-326.
Valle, A., Borges, V.F., Rincones, C. 2000. Principales malezas en cultivos de caña de azúcar en el municipio Unión del estado Falcón, Venezuela. Revista de la Facultad de Agronomía-LUZ, 17(1), 51-62.

Fecha de Recepción: 16 de marzo de 2012

Fecha de Aceptación: 26 de abril de 2013 